\title{
High Expression of H3K27me3 Is an Independent Predictor of Worse Outcome in Patients with Urothelial Carcinoma of Bladder Treated with Radical Cystectomy
}

\author{
Jianye Liu, ${ }^{1,2}$ Yonghong Li, ${ }^{1,2}$ Yiji Liao, ${ }^{1}$ Shijuan Mai, ${ }^{1}$ Zhiling Zhang, ${ }^{1,2}$ Zhouwei Liu, ${ }^{1,2}$ \\ Lijuan Jiang, ${ }^{1,2}$ Yixin Zeng, ${ }^{1}$ Fangjian Zhou, ${ }^{1,2}$ and Dan Xie ${ }^{1,3}$ \\ ${ }^{1}$ State Key Laboratory of Oncology in South China, Sun Yat-sen University Cancer Center, Guangzhou, Guangdong 510060, China \\ ${ }^{2}$ Department of Urology, Sun Yat-sen University Cancer Center, Guangzhou, Guangdong 510060, China \\ ${ }^{3}$ Department of Pathology, Sun Yat-sen University Cancer Center, Guangzhou, Guangdong 510060, China
}

Correspondence should be addressed to Fangjian Zhou; zhouf@sysucc.org.cn and Dan Xie; xiedan@sysucc.org.cn

Received 23 May 2013; Accepted 31 July 2013

Academic Editor: Xin-yuan Guan

Copyright (c) 2013 Jianye Liu et al. This is an open access article distributed under the Creative Commons Attribution License, which permits unrestricted use, distribution, and reproduction in any medium, provided the original work is properly cited.

It has been suggested that trimethylation of lysine 27 on histone $\mathrm{H} 3(\mathrm{H} 3 \mathrm{~K} 27 \mathrm{me} 3)$ is a crucial epigenetic process in tumorigenesis. However, the expression pattern of $\mathrm{H} 3 \mathrm{~K} 27 \mathrm{me} 3$ and its clinicopathological/prognostic significance in urothelial carcinoma of bladder (UCB) are unclear. In this study, upregulated expression of $\mathrm{H} 3 \mathrm{~K} 27 \mathrm{me} 3$ protein was observed in the majority of UCBs by Western blotting. High expression of H3K27me3 was examined by IHC in 59/126 (46.8\%) of UCB tissues and in 18/72 (25.0\%) of normal urothelial bladder epithelial tissues $(P=0.002)$. High expression of H3K27me3 was associated with multifocal tumors and lymph node metastases $(P<0.05)$. Patients with high expression of H3K27me3 had shorter cancer-specific survival (CSS) time than patients with low expression of H3K27me3 $(P<0.001)$. In different subsets of UCB patients, high expression of H3K27me3 was also a prognostic indicator in patients with grade 2 and grade $3, \mathrm{pT} 1, \mathrm{pT} 2$, pT3, and pN- disease $(P<0.05)$. Importantly, expression of H3K27me3 was an independent predictor for CSS $(P<0.001)$ of UCB patients treated with radical cystectomy (RC). Our data suggests that high expression of $\mathrm{H} 3 \mathrm{~K} 27 \mathrm{me} 3$ is an independent molecular marker for predicting poor prognosis of UCB patients treated with RC.

\section{Introduction}

Urothelial carcinoma of bladder (UCB) is one of the major causes of morbidity and mortality in Western countries [1]. Clinically, radical cystectomy (RC) remains the most common treatment for patients with muscle-invasive UCB or for patients with superficial disease that is at high risk of recurrence and progression. Despite advances in surgical technique and an improved understanding of the role of pelvic lymphadenectomy, the 5-year cancer-specific survival (CSS) remains at only $50-60 \%[2,3]$. In addition, while providing important prognostic information on UCB, the currently clinical and pathological variables have a limited ability to predict tumor recurrence, progression, and/or patient survival. The most possibly underlying reason might be the heterogeneous biological properties of UCB. Therefore, the search for specific genes alterations which determine biological nature and behavior of UCB would be of utmost importance to optimize individual therapy. However, such reliable biomarkers are still substantially limited.

It has been reported that epigenetic changes are involved in the silencing of various tumor-suppressor genes, the facilitation of tumorigenesis, and/or the progression of human cancers [4-6]. Histone methylation has been found to play an important role in regulating gene expression and chromatin function [5]. Trimethylation of lysine 27 on histone H3 (H3K27me3), a transcription-suppressor histone modification, is catalyzed by enhancer of zeste homolog 2 (EZH2) [7]. $\mathrm{EZH} 2$, the catalytic subunit of the polycomb repressive complex 2 (PRC2), contributes to the maintenance of cell identity, cell cycle regulation, and tumorigenesis. EZH2 is frequently overexpressed and correlates with poor prognosis in many 
TABLE 1: Association between the expression of H3K27me3 and clinicopathologic features in UCB.

\begin{tabular}{|c|c|c|c|c|}
\hline \multirow{2}{*}{ Characteristic } & \multirow{2}{*}{$\begin{array}{l}\text { Total } \\
\text { cases }\end{array}$} & \multicolumn{3}{|c|}{ H3K27me3 expression (\%) } \\
\hline & & $\begin{array}{c}\text { Low } \\
\text { expression }\end{array}$ & $\begin{array}{c}\text { High } \\
\text { expression }\end{array}$ & $P$ value* \\
\hline Age (years) & & & & 0.955 \\
\hline$\leq 65^{* *}$ & 68 & $36(52.9)$ & $32(47.1)$ & \\
\hline$>65$ & 58 & $31(53.4)$ & $27(46.6)$ & \\
\hline Gender & & & & 0.817 \\
\hline Male & 114 & $61(53.5)$ & $53(46.5)$ & \\
\hline Female & 12 & $6(50.0)$ & $6(50.0)$ & \\
\hline $\begin{array}{l}\text { Tumor } \\
\text { multiplicity }\end{array}$ & & & & 0.010 \\
\hline Unifocal & 49 & $19(38.8)$ & $30(61.2)$ & \\
\hline Multifocal & 77 & $48(62.3)$ & $29(37.7)$ & \\
\hline WHO grade & & & & 0.312 \\
\hline G1 & 24 & $16(66.7)$ & $8(33.3)$ & \\
\hline G2 & 46 & $24(52.2)$ & $22(47.8)$ & \\
\hline G3 & 56 & $27(48.2)$ & $29(51.8)$ & \\
\hline pT status & & & & 0.404 \\
\hline PT1 & 25 & $16(64.0)$ & $9(36.0)$ & \\
\hline PT2 & 55 & $30(54.5)$ & $25(45.5)$ & \\
\hline PT3 & 30 & $15(50.0)$ & $15(50.0)$ & \\
\hline PT4 & 16 & $6(37.5)$ & $10(62.5)$ & \\
\hline pN status & & & & 0.046 \\
\hline $\mathrm{PN}-$ & 105 & $60(57.1)$ & $45(42.9)$ & \\
\hline $\mathrm{PN}+$ & 21 & $7(33.3)$ & $14(66.7)$ & \\
\hline
\end{tabular}

${ }^{*}$ Chi-square test; ${ }^{* *}$ median age; UCB: urothelial carcinoma of bladder.

human cancers [8-12], as well as in UCB [13, 14]. Up to date, however, the protein expression of H3K27me3 in UCB and its associated clinicopathological and prognostic significance have not been investigated. Thus, in the present study, we aimed to investigate the clinical/prognostic implication of H3K27me3 in UCB patients treated with RC.

\section{Material and Methods}

2.1. Patient Information and Tissue Samples. In this study, for analysis of $\mathrm{H} 3 \mathrm{k} 27 \mathrm{me} 3$ protein levels in UCBs by Western blot, 15 pairs of fresh UCB and adjacent morphologically normal bladder tissues underwent RC frozen and stored in liquid nitrogen until further use. In addition, for preparation of the bladder tissue microarray (TMA), 126 patients with UCB that underwent $\mathrm{RC}$ were selected from the surgical pathology archives of the Department of Pathology, Cancer Center, and the First Affiliated Hospital, Sun Yat-Sen University, between 1999 and 2008. Prior patients' consent and approval from the Institutional Research Ethics Committee of Sun Yat-Sen University Cancer Center were obtained for the use of these clinical materials for research purposes. Clinical information on the samples is summarized in Table 1. The tumor specimens were recruited from paraffin blocks of
126 primary UCBs. Seventy-two cases of normal bladder mucosa from adjacent nonneoplastic bladder tissue of the same UCB patients, in paraffin blocks, were also obtained. None of the UCB patients included in this study had received preoperative radiation or chemotherapy. Tumor grade and stage were defined according to the criteria of the WHO and the 6th edition of the pTNM classification of the International Union Against Cancer (UICC, 2002).

2.2. Western Blotting Analysis. Equal amounts of whole cell and tissue lysates were resolved by SDS-polyacrylamide gel electrophoresis and electrotransferred onto a polyvinylidene difluoride membrane (Pall Corp., Port Washington, NY, USA). The tissues were then incubated with primary rabbit monoclonal antibodies against H3K27me3 (1:1000 dilution; Cell Signaling Technology, Beverly, MA, USA). The immunoreactive signals were detected with an enhanced chemiluminescence kit (Amersham Biosciences, Uppsala, Sweden). The procedures were conducted in accordance with the manufacturers' instructions. GAPDH antibody $(1: 2000$ dilution; Sigma, St. Louis, MO, USA) was used as the loading control.

2.3. Construction of TMAs. The TMA was constructed according to a method described previously [15]. In brief, the paraffin-embedded tissue blocks and the corresponding histological hematoxylin-and-eosin- (H\&E-) stained slides were overlaid for tissue TMA sampling. Duplicates of $0.6 \mathrm{~mm}$ diameter cylinders were punched from representative tumor areas of individual donor tissue blocks and reembedded into a recipient paraffin block at a defined position, using a tissue arraying instrument (Beecher Instruments, Silver Spring, MD, USA). In our constructed bladder tissue TMA, 3 cores of sample were selected from each primary UCB and normal bladder tissue. Multiple sections ( $5 \mu \mathrm{m}$ thick) were cut from the TMA block and mounted on microscope slides.

2.4. Immunohistochemistry (IHC). IHC studies were performed using a standard streptavidin-biotin-peroxidase complex method. In brief, TMA sections were deparaffinized and rehydrated. Endogenous peroxidase activity was blocked with $0.3 \%$ hydrogen peroxide for $15 \mathrm{~min}$. For antigen retrieval, tissue slides were boiled in $10 \mathrm{mM}$ citrate buffer ( $\mathrm{pH}$ 6.0) and microwave-treated for $10 \mathrm{~min}(\mathrm{H} 3 \mathrm{~K} 27 \mathrm{me} 3)$ or in Tris (hydroxymethyl) aminomethane-EDTA buffer ( $\mathrm{pH} 8.0)$ in a pressure cooker for $12 \mathrm{~min}$ (EZH2). Nonspecific binding was blocked with $10 \%$ normal rabbit serum for $20 \mathrm{~min}$. The tissue slides were incubated with anti-H3K27me3 (1:50; Abcam, Cambridge, MA, USA) or anti-EZH2 (1:100; BD Transduction Laboratories, Franklin Lakes, NJ, USA) for $60 \mathrm{~min}$ at $37^{\circ} \mathrm{C}$ in a moist chamber. Subsequently, the slides were sequentially incubated with biotinylated rabbit antimouse immunoglobulin at a concentration of 1:100 for $30 \mathrm{~min}$ at $37^{\circ} \mathrm{C}$ and then reacted with a streptavidin-peroxidase conjugate for $30 \mathrm{~min}$ at $37^{\circ} \mathrm{C}$ and $3^{\prime}-3^{\prime}$ diaminobenzidine as a chromogen substrate. The nucleus was counterstained using Meyer's hematoxylin. A negative control was obtained by replacing the primary antibody with a normal murine 
immunoglobulin. Known immunostaining positive slides were used as positive controls.

To evaluate of the H3K27me3 and EZH2 IHC staining in different bladder tissues, the nuclear pattern of $\mathrm{H} 3 \mathrm{~K} 27 \mathrm{me} 3$ and EZH2 in bladder tissues was recorded as positive expression. Nuclear immunoreactivity scores for H3K27me3 [16, 17] and EZH2 $[8,12,18]$ proteins were calculated using previously validated respective scoring systems, respectively. For $\mathrm{H} 3 \mathrm{~K} 27 \mathrm{me} 3$, the system used calculated the percentage of nuclei that stained positive for the $\mathrm{H} 3 \mathrm{~K} 27 \mathrm{me} 3$ protein in multiples of 10. As the frequency of the percentage of positively stained cells in all tumor samples assessed for $\mathrm{H} 3 \mathrm{~K} 27 \mathrm{me} 3$ was almost normally distributed and ranged from $0 \%$ to $100 \%$ and the median value was $50 \%$, a $50 \%$ cut-off value was used to, categorize samples into high and low expression levels $[16,17]$. For EZH2, the system scored nuclear EZH2 expression by recording the percentage of nuclei with $\mathrm{EZH} 2$ immunoreactivity and classified samples into two groups: low expression, where there was $<50 \%$ positive cells; and high expression, when $\geqq 50 \%$ of the cells showed nuclear immunoreactivity [8, 12, 18]. H3K27me3 and EZH2 expression levels were assessed by pathologists who were blinded to the clinicopathological data.

2.5. Statistical Analysis. All statistical analyses were carried out using the SPSS v. 13.0 statistical software packages (SPSS, Chicago, IL, USA). The relationship between H3K27me3 expression and clinicopathological characteristics was analyzed by the Chi-square test. Survival curves were plotted by the Kaplan-Meier method and compared using the logrank test. Survival data were evaluated using univariate and multivariate Cox regression analyses. $P$ values of less than 0.05 were considered to indicate statistical significance.

\section{Results}

3.1. Expression Patterns of H3K27me3 in UCB Cells and Tissues by Western Blotting. To investigate the protein levels of $\mathrm{H} 3 \mathrm{~K} 27 \mathrm{me} 3$ in UCB tissues, protein expression of H3K27me3 in 15 pairs of primary UCB and adjacent normal bladder specimens was analyzed using Western blotting. As shown in Figure 1, a total of 12 out of $15(80.0 \%)$ UCB tissues samples had upregulated levels of $\mathrm{H} 3 \mathrm{~K} 27 \mathrm{me} 3$ expression, compared with their adjacent normal bladder tissues. The results revealed that $\mathrm{H} 3 \mathrm{~K} 27 \mathrm{me} 3$ was upregulated at the protein level, in clinical tissue samples of UCB.

3.2. The Expression Dynamics of H3K27me3 Examined by IHC in Bladder Tissue TMA. In IHC study, immunoreactivity of $\mathrm{H} 3 \mathrm{~K} 27 \mathrm{~m} 3$ was observed primarily in the cell nuclei, though occasionally yellowish brown granules could also be seen in the cytoplasm (Figure 2). H3K27me3 expression could be evaluated informatively in 113/126 of UCB tissues and $61 / 72$ of normal bladder tissues. The noninformative samples included unrepresentative samples, samples with too few tumor cells ( $<300$ cells per case), and lost samples. For the noninformative TMA samples, IHC staining was replaced and performed by using whole tissue slides. By using
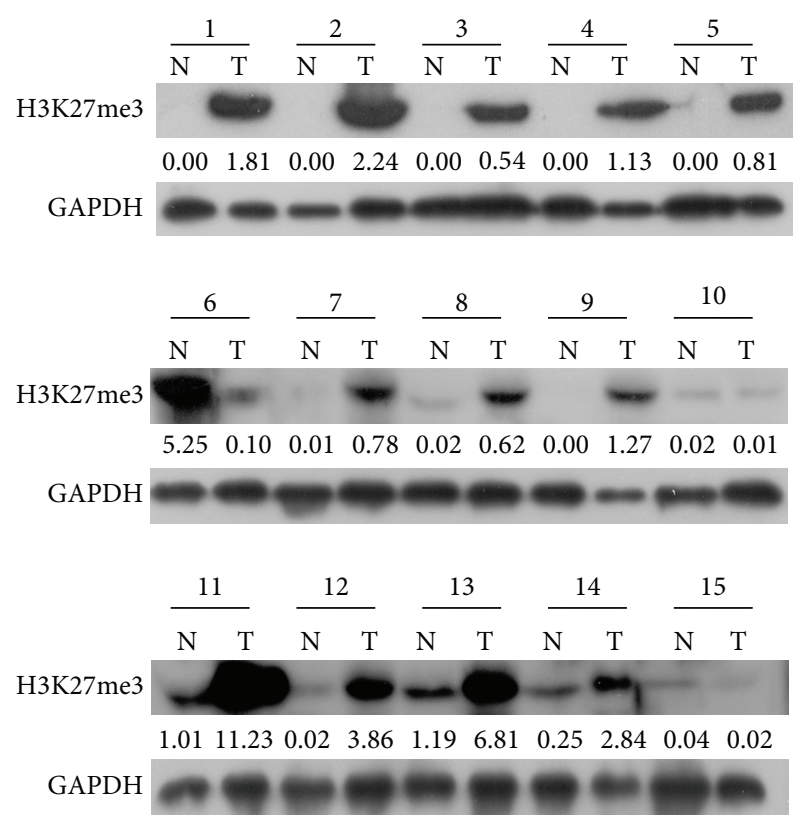

FIGURE 1: The expression of H3K27me3 protein in UCB tissues by Western bloting analysis. Upregulated expression of $\mathrm{H} 3 \mathrm{~K} 27 \mathrm{me} 3$ was observed in 12/15 of UCB tissues compared to adjacent normal urothelial mucosal tissues. Expression levels were normalized with GAPDH.

the criteria (cutoff score) for $\mathrm{H} 3 \mathrm{~K} 27 \mathrm{me} 3$ staining described before, high expression of $\mathrm{H} 3 \mathrm{~K} 27 \mathrm{me} 3$ was examined in 59/126 $(46.8 \%)$ of UCB and in $18 / 72(25.0 \%)$ of normal urothelial bladder epithelial tissues $(P=0.002)$. The rates of high expression of $\mathrm{H} 3 \mathrm{~K} 27 \mathrm{me} 3$ in UCBs with respect to several standard clinicopathologic features were detailed in Table 1. Correlation analysis demonstrated that high expression of H3K27me3 in UCBs was positively correlated with tumor multiplicity and $\mathrm{N}$ classification $(P<0.05$, Table 1$)$. There was no significant association between $\mathrm{H} 3 \mathrm{~K} 27 \mathrm{me} 3$ expression and other clinicopathologic features, such as patient gender, age, tumor grade, and T classification $(P>0.05$, Table 1$)$.

3.3. Relationship between Clinicopathologic Variables, H3K27me3 Expression and UCB Patient Survival: Univariate Survival Analysis. In univariate survival analyses, cumulative survival curves were calculated according to the KaplanMeier method. Differences in survival times were assessed with the log-rank test. Kaplan-Meier analysis showed a significant effect of certain clinical pathologic prognostic variables, such as tumor multiplicity $(P=0.026)$, tumor pT status $(P<0.001)$, and tumor $\mathrm{pN}$ status $(P<0.001)$ on patient survival (Table 2). Assessment of UCB patient survival also revealed that high expression of $\mathrm{H} 3 \mathrm{~K} 27 \mathrm{me} 3$ was correlated significantly with poor cancer-specific survival (CSS, $P<0.001$; Figure 3; Table 2). Additionally, survival analysis was done with regard to $\mathrm{H} 3 \mathrm{~K} 27 \mathrm{me} 3$ expression in subsets of patients with different tumor histopathologic grades, $\mathrm{pT}$ and $\mathrm{pN}$ stages. The results showed that high expression of $\mathrm{H} 3 \mathrm{~K} 27 \mathrm{me} 3$ was also a prognostic factor in UCB patients in grade $2(P<0.001)$ and grade $3(P=0.011)$, 


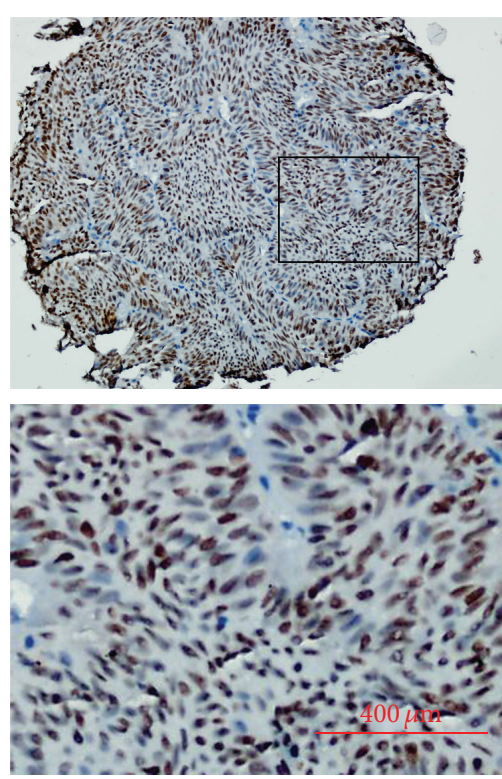

(a)
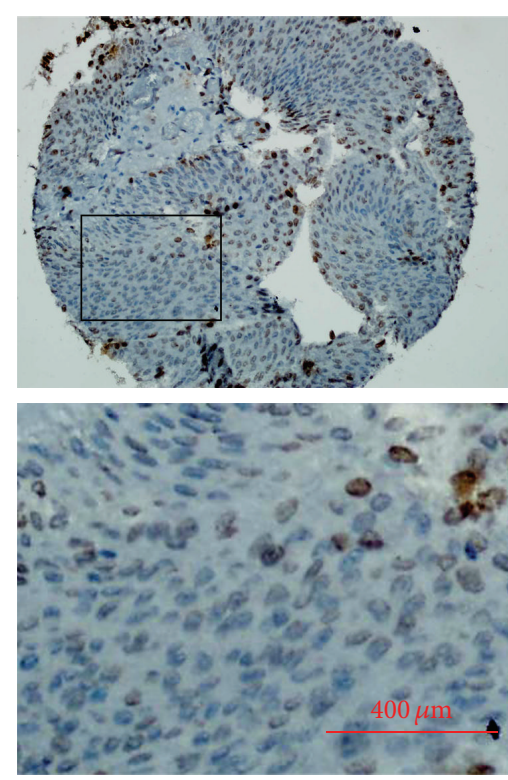

(b)
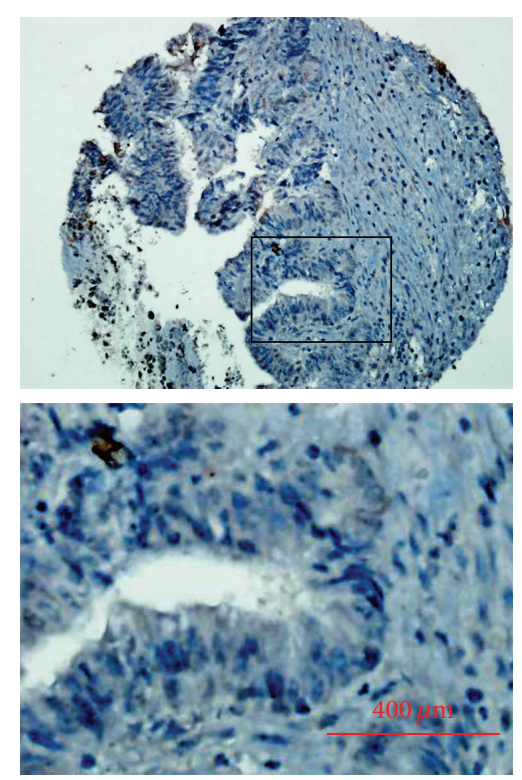

(c)

FIGURE 2: The expression of H3K27me3 in UCB and adjacent normal bladder tissues by IHC. (a) High expression of H3K27me3 was shown in a UCB case (case 25), in which more than $80 \%$ of carcinoma cells revealed positive staining of H3K27me3 protein in nuclei (100X). (b) Another UCB case (case 34) demonstrated low expression of $\mathrm{H} 3 \mathrm{~K} 27 \mathrm{me} 3$, in which less than $30 \%$ of carcinoma cells showed positive staining of H3K27me3 protein in nuclei (100X). (c) Adjacent normal bladder urothelial mucosal tissue showed nearly negative expression of H3K27me3 protein (100X). The lower panels indicated the higher magnification (400X) from the area of the box in the upper panels.

pT1 $(P=0.027)$, pT2 $(P=0.018)$, pT3 $(P=0.001)$, and pN$(P<0.001 ;$ Figure 3).

\subsection{Independent Prognostic Factors of UCB: Multivariate} Survival Analysis. As the variables observed to have prognostic influence by univariate analysis may be covariate, the expression of $\mathrm{H} 3 \mathrm{~K} 27 \mathrm{me} 3$ and other clinicopathological features that were significant in the univariate analysis (tumor multiplicity, T classification, and N classification) were examined by a multivariate analysis (Table 3 ). We found that the high expression of H3K27me3 was an independent risk factor for adverse CSS (hazards ratio: 4.973; 95\% confidence interval: 2.137-11.569; $P<0.001$ ). Of the other variables, $\mathrm{N}$ classification also was found to be an independent prognostic predictor for CSS (Table 3).

3.5. Correlation between the Expression of H3K27me3 and $E Z H 2$ in UCB. For EZH2 staining, using the criteria described above, the high expression of EZH2 was observed in $67 / 126(53.2 \%)$ of tissue samples of UCB, while the other 59 cases showed low EZH2 expression levels. Thus, we further evaluated the relationship between the expression of $\mathrm{H} 3 \mathrm{~K} 27 \mathrm{me} 3$ and EZH2 in a cohort. The results showed a positive correlation between the expression levels of H3K27me3 and EZH2 (Figures 4(a) and 4(b)). For the 59 UCB cases with high $\mathrm{H} 3 \mathrm{~K} 27 \mathrm{me} 3$ expression, $61.0 \%$ of carcinoma cells on average stained positive for EZH2 protein. This percentage was significantly higher than that of the 67 UCBs with low expression levels of H3K27me3 (46.3\%; $P=0.003$, independent sample $t$ test; Figure 4(c)).

\section{Discussion}

Clinically, approximately $50 \%$ to $60 \%$ of patients diagnosed with muscle-invasive UCB will develop metastatic progression after local therapy with curative intent, resulting in approximately 12,000 deaths annually $[2,3]$. Although current pTNM staging and histopathological grading systems have been established and are useful prognostic indicators for UCB after local therapy [19], these approaches are in the extent that they can provide information regarding patient prognosis and optimal treatment approaches. Patients with the same stage and/or grade of UCB treated with $\mathrm{RC}$ often display considerable variability in rates of disease recurrence and survival $[20,21]$. Therefore, there is a need for new objective strategies that can effectively distinguish between patients with favorable and unfavorable prognoses. Individuals that are identified to have different prognoses by molecular biomarkers prior to surgery can have prolonged survival times with the addition of more effective adjuvant therapies [20]. Although UCB has been widely studied, the identification of specific genetic alterations associated with UCB tumorigenic processes and their clinical/prognostic significance remains substantially limited. Thus, further work is clearly needed to develop appropriate biomarkers.

Histone modifications are epigenetic mechanisms that play crucial roles in tumorigenesis [4]. One such modification, the trimethylation of $\mathrm{H} 3 \mathrm{~K} 27$, is mediated by proteins in the polycomb group family of genes. These were originally identified as genes that suppressed the development of extra sex combs in Drosophila [22]. It has been suggested that the maintenance of the $\mathrm{H} 3 \mathrm{~K} 27$ me3 epigenetic mark during 


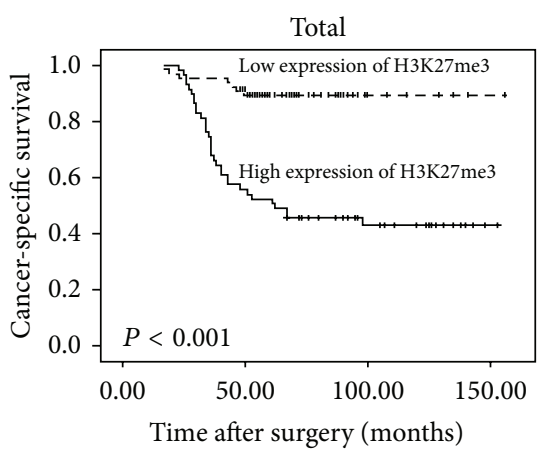

(a)

G3

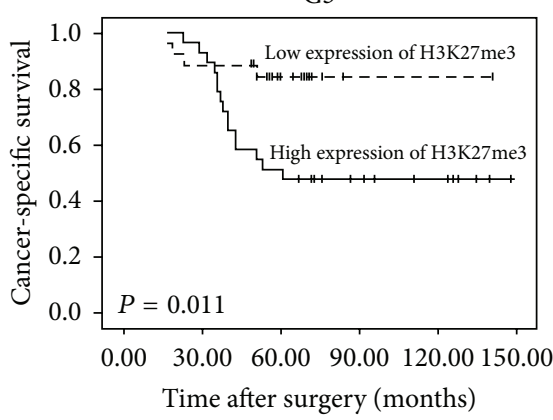

(d)

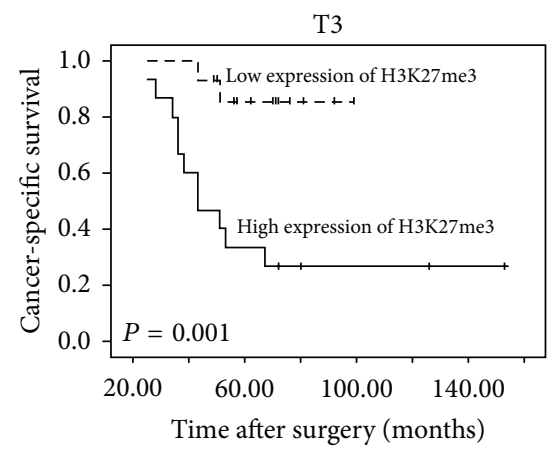

$(\mathrm{g})$

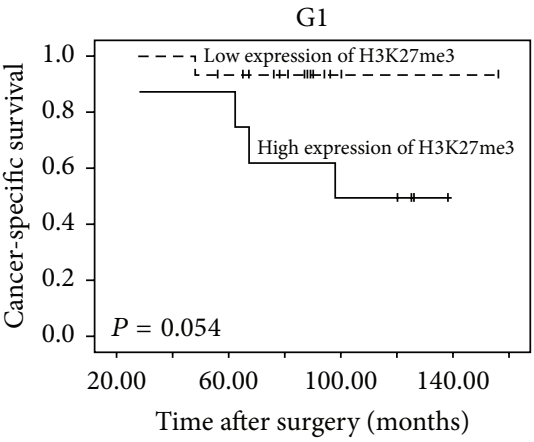

(b)

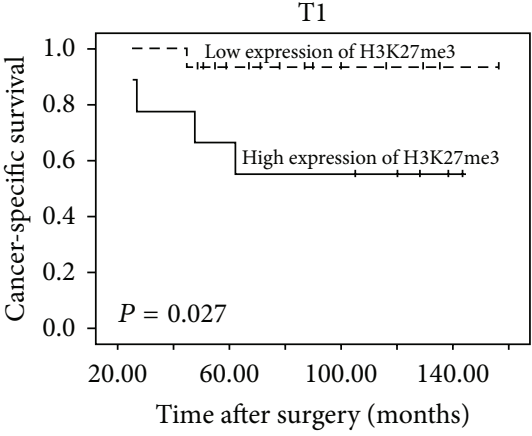

(e)

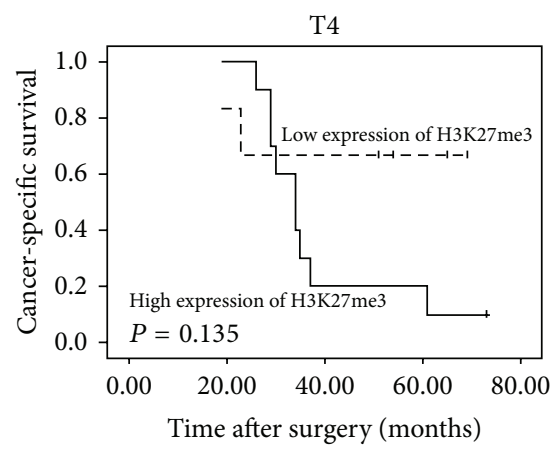

(h)

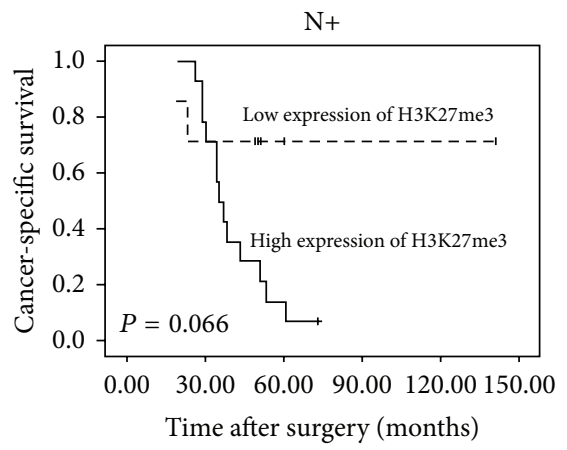

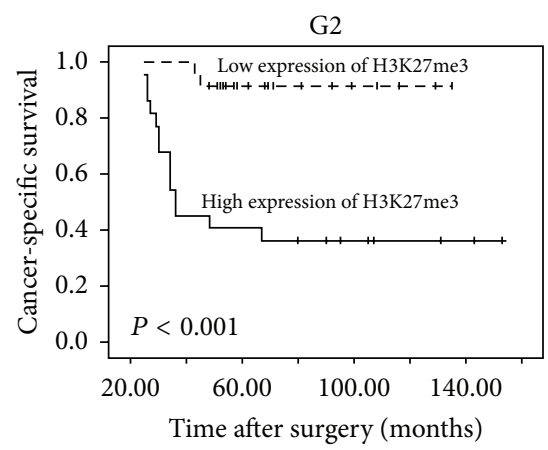

(c)

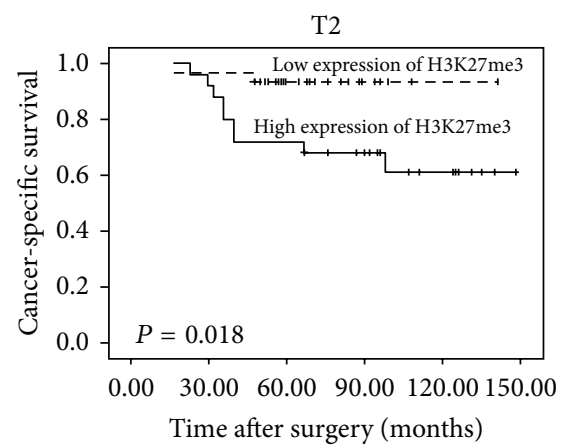

(f)

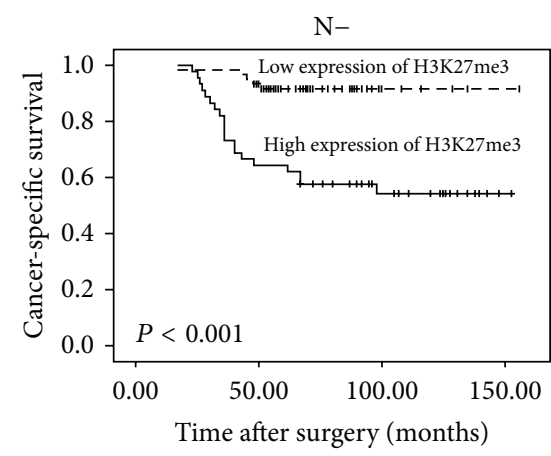

(i)

(j)

FIgURE 3: Kaplan-Meier survival analysis of H3K27me3 expression in patients with UCB (log-rank test). Total, probability of survival of all patients with UCB: low expression (dashed line), $n=67$; high expression (solid line), $n=59$. G1, probability of survival of G1 patients with UCB: low expression (dashed line), $n=16$; high expression (solid line), $n=8$. G2, probability of survival of G2 patients with UCB: low expression (dashed line), $n=24$; high expression (solid line), $n=22$. G3, probability of survival of G3 patients with UCB: low expression (dashed line), $n=27$; high expression (solid line), $n=29$. pT1, probability of survival of pT1 patients with UCB: low expression (dashed line), $n=16$; high expression (solid line), $n=9$. pT2, probability of survival of pT2 patients with UCB: low expression (dashed line), $n=30$; high expression (solid line), $n=25$. pT3, probability of survival of pT3 patients with UCB: low expression (dashed line), $n=15$; high expression (solid line), $n=15$. pT4, probability of survival of pT4 patients with UCB: low expression (dashed line), $n=6$; high expression (solid line), $n=10$. $p N-$, probability of survival of $\mathrm{pN}$ - patients with UCB: low expression (dashed line), $n=60$; high expression (solid line), $n=45$. $p N+$, probability of survival of $\mathrm{pN}+$ patients with UCB: low expression (dashed line), $n=7$; high expression (solid line), $n=14$. 


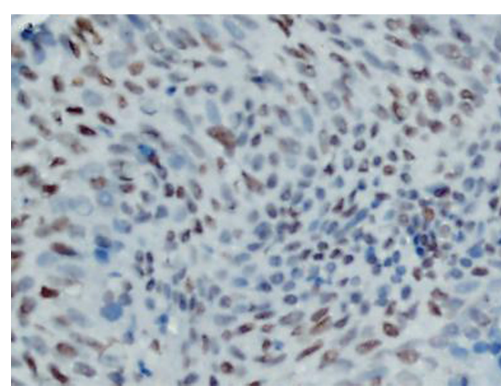

(a)

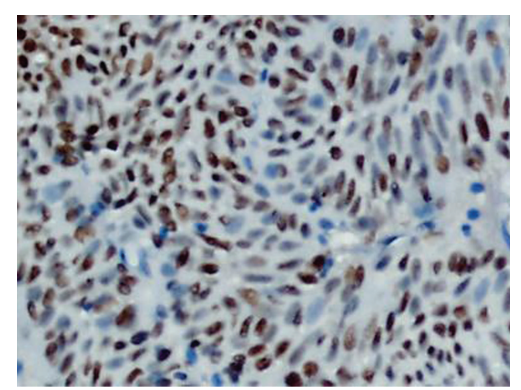

(b)

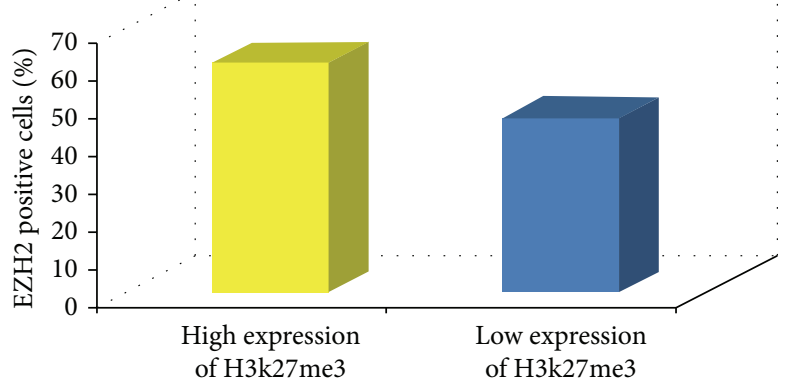

(c)

FIGURE 4: Correlation between expressions of H3K27me3 and EZH2 in UCB tissues. (a) High expression for EZH2 was observed in a UCB (case 86), in which more than 70\% of tumor cells showed nuclear positive staining of EZH2 protein (400X). (b) Overexpression of H3K27me3 was examined in the same UCB case 86 (400X). (c) In 59 UCB cases with high expression of H3K27me3, an average of $61.0 \%$ of the UCB cells stained positive with EZH2, a percentage of cancer cells that was significantly larger than that (46.3\%) in 67 UCBs with low expression of H3K27me3 ( $P=0.003$, independent sample $t$ test).

cell division is pivotal for normal embryogenesis and cell identity [23]. The methylation of H3K27 mediated by EZH2 has been implicated in the aggressive phenotype of cancer cells through the repression of a panel of tumor suppressor genes $[24,25]$. The loss of function of these genes, in turn, locks stem/precursor cells into abnormal clonal expansion which begins the process of neoplastic initiation $[26,27]$. This might provide a possible explanation for the observed association between $\mathrm{H} 3 \mathrm{~K} 27 \mathrm{me} 3$ expression and advanced pN stage in UCB found in this current study. Moreover, an imbalance in H3K27 methylation owing to overexpression of EZH2 has been implicated in metastatic prostate and aggressive breast cancers [11,28], in which a highly significant overlap between PRC2- and H3K27me3-occupied genes was observed [28]. To determine whether there was a potential correlation between the expression of EZH2 and H3K27me3 in UCB, we evaluated the expression status of these 2 proteins by IHC in the same cohort of cases. Our results demonstrated that the expression level of EZH2 in the high H3K27me3 expression group was significantly higher than that in the low H3K27me3 expression group, which supported the view that the upregulated expression of H3K27me3 in UCBs might be caused, at least in part, by the increased expression of EZH2. Moreover, H3K27 trimethylation has been shown to be correlated with the development and/or progression of different human cancers [5]. To date, however, the expression dynamics of H3K27me3 in UCB and its potential impact on UCB tumorigenesis and/or prognosis have not been elucidated.
In the present study, we reported, for the first time, the clinical significance of H3K27me3 in UCB. This is also the first study that aimed to evaluate the possibility of using $\mathrm{H} 3 \mathrm{~K} 27 \mathrm{me} 3$ as a clinically potential indicator for disease progression as well as a prognostic marker for patient survival in UCB. Our results, from Western blot analysis, indicated that the expression of endogenous H3K27me3 was upregulated in the majority of UCB tissue samples. Next, the expression dynamics of H3K27me3 was examined by IHC in a large cohort of UCB tissues samples taken from patients who underwent RC. These were analyzed using a bladder tissue TMA with complete follow-up data of each patient. Our results demonstrated that the frequency of high H3K27me3 expression in UCB tissues was significantly larger than that in nonneoplastic bladder epithelial tissues. These findings suggest that the upregulated expression of H3K27me3 may provide a selective advantage in UCB tumorigenic processes. Furthermore, we showed that the expression level of $\mathrm{H} 3 \mathrm{~K} 27 \mathrm{me} 3$ protein significantly correlated with the clinical characteristics of UCB, including tumor multiplicity, $\mathrm{N}$ classification, and patient prognosis. These findings were similar to those of other studies $[18,29]$, in which the H3K27me3 protein was found to be frequently overexpressed in esophageal and hepatocellular carcinomas and positively correlated with tumor aggressiveness and/or advanced clinical stage. Taken together, these data suggest that the upregulation of H3K27me3 may facilitate the invasive/metastatic phenotypes of different types of human cancers, including UCB. 
TABLE 2: Univariate analysis of $\mathrm{H} 3 \mathrm{~K} 27 \mathrm{~m} 3$ expression and various clinicopathological parameters in 126 patients with UCB.

\begin{tabular}{|c|c|c|c|}
\hline Characteristic & Total cases & RR $(95 \%$ CI $)$ & $P$ value \\
\hline Age, years & & & 0.786 \\
\hline$\leq 65^{*}$ & 68 & 1 & \\
\hline$>65$ & 58 & $1.090(0.586-2.027)$ & \\
\hline Gender & & & 0.675 \\
\hline Male & 114 & 1 & \\
\hline Female & 12 & $0.777(0.239-2.523)$ & \\
\hline Tumor multiplicity & & & 0.026 \\
\hline Unifocal & 49 & 1 & \\
\hline Multifocal & 77 & $2.025(1.088-3.771)$ & \\
\hline WHO grade & & & 0.330 \\
\hline G1 & 24 & 1 & \\
\hline G2 & 46 & $2.069(0.757-5.655)$ & \\
\hline G3 & 56 & $2.016(0.751-5.412)$ & \\
\hline pT status & & & $<0.001$ \\
\hline PT1 & 25 & 1 & \\
\hline PT2 & 55 & $1.047(0.363-3.017)$ & \\
\hline PT3 & 30 & $2.561(0.908-7.219)$ & \\
\hline PT4 & 16 & $6.577(2.250-19.227)$ & \\
\hline $\mathrm{pN}$ status & & & $<0.001$ \\
\hline $\mathrm{PN}-$ & 105 & 1 & \\
\hline $\mathrm{PN}+$ & 21 & 4.957 (2.573-9.549) & \\
\hline $\mathrm{H} 3 \mathrm{~K} 27 \mathrm{me} 3$ & & & $<0.001$ \\
\hline Low expression & 67 & 1 & \\
\hline High expression & 59 & $6.476(2.857-14.676)$ & \\
\hline
\end{tabular}

${ }^{*}$ Median age; RR: relative risk; CI: confidence interval; UCB: urothelial carcinoma of bladder.

TABLE 3: Cox multivariate analyses of prognostic factors on survival.

\begin{tabular}{lccc}
\hline Variable & Hazards ratio & $95 \%$ CI & $P$ value \\
\hline $\begin{array}{l}\text { Tumor multiplicity (unifocal } \\
\text { versus multifocal) }\end{array}$ & 1.352 & $0.704-2.595$ & 0.365 \\
pT status (PT1 versus PT2 & 1.480 & $0.893-2.450$ & 0.128 \\
versus PT3 versus PT4) & & $0.816-5.751$ & 0.021 \\
pN status (PN- versus PN+) & 2.166 & $0.137-11.569$ & $<0.001$ \\
\hline H3K27me3 (low versus high) & 4.973 & &
\end{tabular}

CI: confidence interval.

\section{Conclusions}

In this study, we reported for the first time that H3K27me3 expression was upregulated in clinical UCB tissues, and high expression of $\mathrm{H} 3 \mathrm{k} 27 \mathrm{me} 3$ was associated closely with a more malignant clinical feature and/or poor prognosis of UCB patients. Our results suggest that $\mathrm{H} 3 \mathrm{~K} 27 \mathrm{me} 3$ overexpression might be useful as a prognostic factor for UCB patients. Apparently, a further understanding of the molecular mechanism by which $\mathrm{H} 3 \mathrm{~K} 27 \mathrm{me} 3$ is involved in cancer cell initiation, proliferation, and/or transformation in human UCB would help in the discovery of novel targeted agents and might also lead to the development of new approaches for effective therapy of human UCB.

\section{Conflict of Interests}

The authors declare that they have no conflict of interests.

\section{Authors' Contribution}

Jianye Liu and Yonghong Li contributed equally to the work.

\section{Acknowledgments}

This study was supported by research Grants from the National Nature Science Foundation of China (nos. 81225018 and 81172340), the 973 Project of China (nos. 2010CB912802 and 2010CB529404), and the Ph.D. Programs Foundation of Ministry of Education of China (no. 20110171110078).

\section{References}

[1] C. Pelucchi, C. Bosetti, E. Negri, M. Malvezzi, and C. La Vecchia, "Mechanisms of disease: the epidemiology of bladder cancer," Nature Clinical Practice Urology, vol. 3, no. 6, pp. 327-340, 2006.

[2] M. S. Cookson, "The surgical management of muscle invasive bladder cancer: a contemporary review," Seminars in Radiation Oncology, vol. 15, no. 1, pp. 10-18, 2005.

[3] J. P. Stein, G. Lieskovsky, R. Cote et al., "Radical cystectomy in the treatment of invasive bladder cancer: long-term results in 1,054 patients," Journal of Clinical Oncology, vol. 19, no. 3, pp. 666-675, 2001.

[4] M. Esteller, "Molecular origins of cancer: epigenetics in cancer," The New England Journal of Medicine, vol. 358, no. 11, pp. 11481096, 2008.

[5] B. D. Strahl and C. D. Allis, "The language of covalent histone modifications," Nature, vol. 403, no. 6765, pp. 41-45, 2000.

[6] A. H. Lund and M. Van Lohuizen, "Epigenetics and cancer," Genes and Development, vol. 18, no. 19, pp. 2315-2335, 2004.

[7] R. Cao, L. Wang, H. Wang et al., "Role of histone H3 lysine 27 methylation in polycomb-group silencing," Science, vol. 298, no. 5595, pp. 1039-1043, 2002.

[8] Y. Matsukawa, S. Semba, H. Kato, A. Ito, K. Yanagihara, and H. Yokozaki, "Expression of the enhancer of zeste homolog 2 is correlated with poor prognosis in human gastric cancer," Cancer Science, vol. 97, no. 6, pp. 484-491, 2006.

[9] K. Mimori, K. Ogawa, M. Okamoto, T. Sudo, H. Inoue, and M. Mori, "Clinical significance of enhancer of zeste homolog 2 expression in colorectal cancer cases," European Journal of Surgical Oncology, vol. 31, no. 4, pp. 376-380, 2005.

[10] T. Sudo, T. Utsunomiya, K. Mimori et al., "Clinicopathological significance of EZH2 mRNA expression in patients with hepatocellular carcinoma," British Journal of Cancer, vol. 92, no. 9, pp. 1754-1758, 2005.

[11] K. Collett, G. E. Eide, J. Arnes et al., "Expression of enhancer of zeste homologue 2 is significantly associated with increased tumor cell proliferation and is a marker of aggressive breast cancer," Clinical Cancer Research, vol. 12, no. 4, pp. 1168-1174, 2006.

[12] K. Kidani, M. Osaki, T. Tamura et al., "High expression of EZH2 is associated with tumor proliferation and prognosis in human oral squamous cell carcinomas," Oral Oncology, vol. 45, no. 1, pp. 39-46, 2009. 
[13] J. D. Raman, N. P. Mongan, S. K. Tickoo, S. A. Boorjian, D. S. Scherr, and L. J. Gudas, "Increased expression of the polycomb group gene, EZH2, in transitional cell carcinoma of the bladder," Clinical Cancer Research, vol. 11, no. 24, pp. 8570-8576, 2005.

[14] H. Wang, R. Albadine, A. Magheli et al., "Increased EZH2 protein expression is associated with invasive urothelial carcinoma of the bladder," Urologic Oncology, vol. 30, no. 4, pp. 428-433, 2012.

[15] J. Southgate, K. A. R. Hutton, D. F. M. Thomas, and L. K. Trejdosiewicz, "Normal human urothelial cells in vitro: proliferation and induction of stratification," Laboratory Investigation, vol. 71, no. 4, pp. 583-594, 1994.

[16] D. Xie, J. S. T. Sham, W.-F. Zeng et al., "Heterogeneous expression and association of $\beta$-catenin, p16 and c-myc in multistage colorectal tumorigenesis and progression detected by tissue microarray," International Journal of Cancer, vol. 107, no. 6, pp. 896-902, 2003.

[17] Y. Wei, W. Xia, Z. Zhang et al., "Loss of trimethylation at lysine 27 of histone $\mathrm{H} 3$ is a predictor of poor outcome in breast, ovarian, and pancreatic cancers," Molecular Carcinogenesis, vol. 47, no. 9, pp. 701-706, 2008.

[18] C. Tzao, H.-J. Tung, J.-S. Jin et al., "Prognostic significance of global histone modifications in resected squamous cell carcinoma of the esophagus," Modern Pathology, vol. 22, no. 2, pp. 252-260, 2009.

[19] M. K. Gospodarowicz, "Staging of bladder cancer," Seminars in Surgical Oncology, vol. 10, no. 1, pp. 51-59, 1994.

[20] B. P. Schrier, M. P. Hollander, B. W. G. Van Rhijn, L. A. L. M. Kiemeney, and J. A. Witjes, "Prognosis of muscle-invasive bladder cancer: difference between primary and progressive tumours and implications for therapy," European Urology, vol. 45, no. 3, pp. 292-296, 2004.

[21] S. A. Hussain and N. D. James, "Molecular markers in bladder cancer," Seminars in Radiation Oncology, vol. 15, no. 1, pp. 3-9, 2005.

[22] E. B. Lewis, "A gene complex controlling segmentation in Drosophila," Nature, vol. 276, no. 5688, pp. 565-570, 1978.

[23] K. H. Hansen, A. P. Bracken, D. Pasini et al., "A model for transmission of the H3K27me3 epigenetic mark," Nature Cell Biology, vol. 10, no. 11, pp. 1291-1300, 2008.

[24] T. Tonini, G. D’Andrilli, A. Fucito, L. Gaspa, and L. Bagella, "Importance of Ezh2 polycomb protein in tumorigenesis process interfering with the pathway of growth suppressive key elements," Journal of Cellular Physiology, vol. 214, no. 2, pp. 295300, 2008.

[25] Y. Schlesinger, R. Straussman, I. Keshet et al., "Polycombmediated methylation on Lys27 of histone $\mathrm{H} 3$ pre-marks genes for de novo methylation in cancer," Nature Genetics, vol. 39, no. 2, pp. 232-236, 2007.

[26] M. Widschwendter, H. Fiegl, D. Egle et al., "Epigenetic stem cell signature in cancer," Nature Genetics, vol. 39, no. 2, pp. 157-158, 2007.

[27] M. V. Brock, J. G. Herman, and S. B. Baylin, "Cancer as a manifestation of aberrant chromatin structure," Cancer Journal, vol. 13, no. 1, pp. 3-8, 2007.

[28] J. Yu, J. Yu, D. R. Rhodes et al., "A polycomb repression signature in metastatic prostate cancer predicts cancer outcome," Cancer Research, vol. 67, no. 22, pp. 10657-10663, 2007.

[29] T.-Y. Lu, C.-F. Kao, C.-T. Lin et al., "DNA methylation and histone modification regulate silencing of OPG during tumor progression," Journal of Cellular Biochemistry, vol. 108, no. 1, pp. 315-325, 2009. 


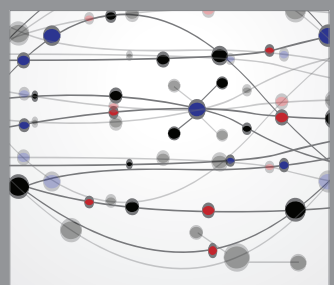

The Scientific World Journal
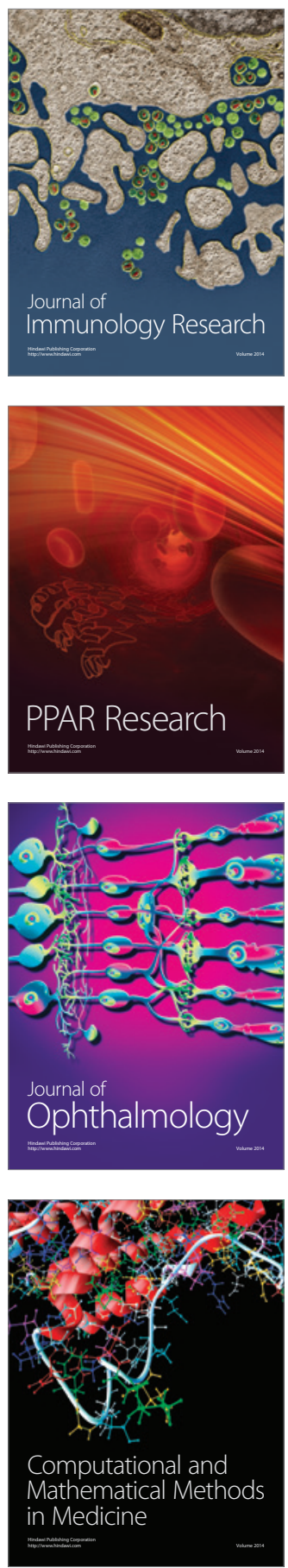

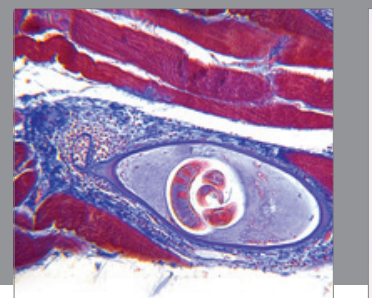

Gastroenterology

Research and Practice
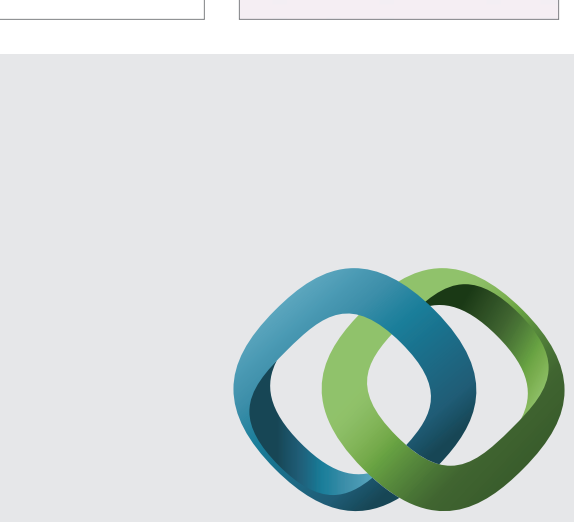

\section{Hindawi}

Submit your manuscripts at

http://www.hindawi.com
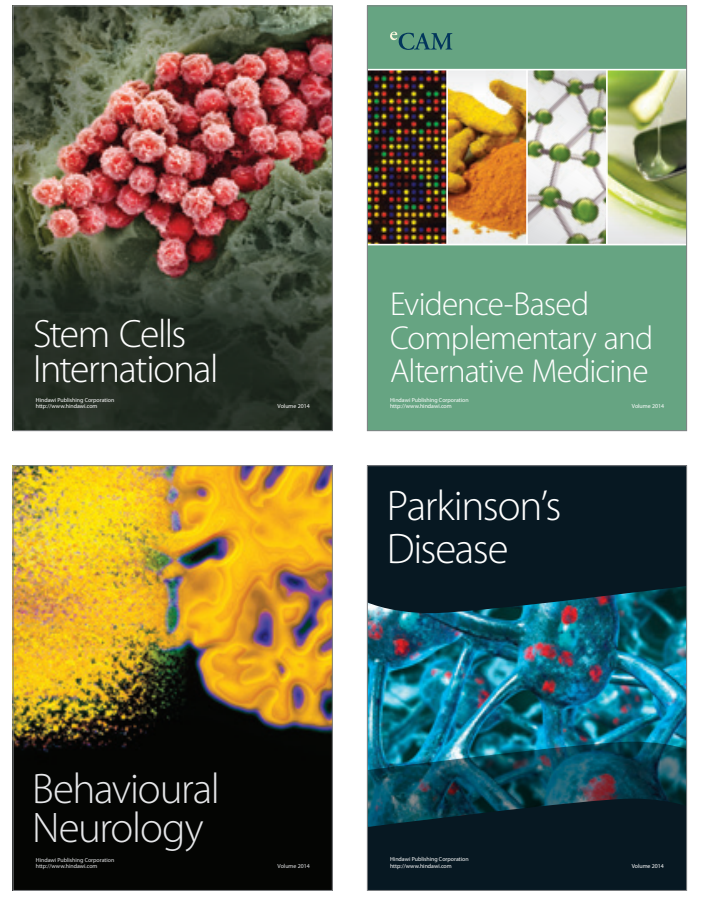
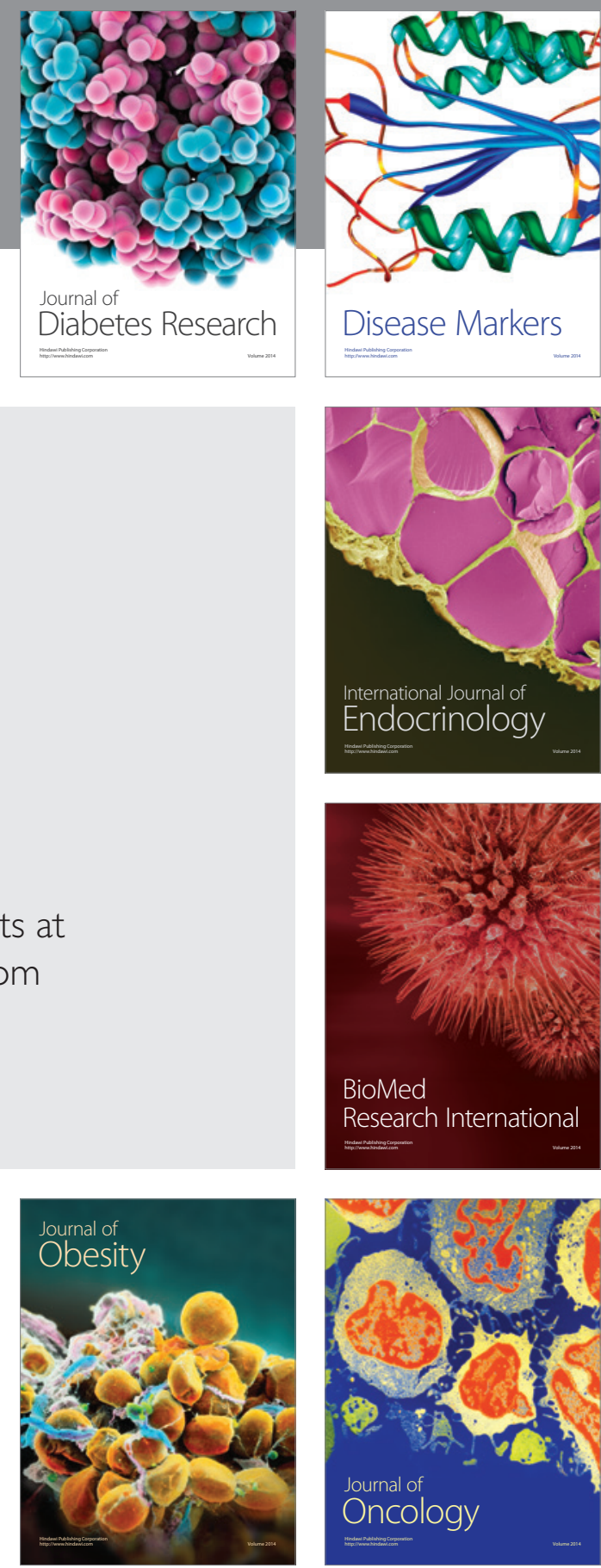

Disease Markers
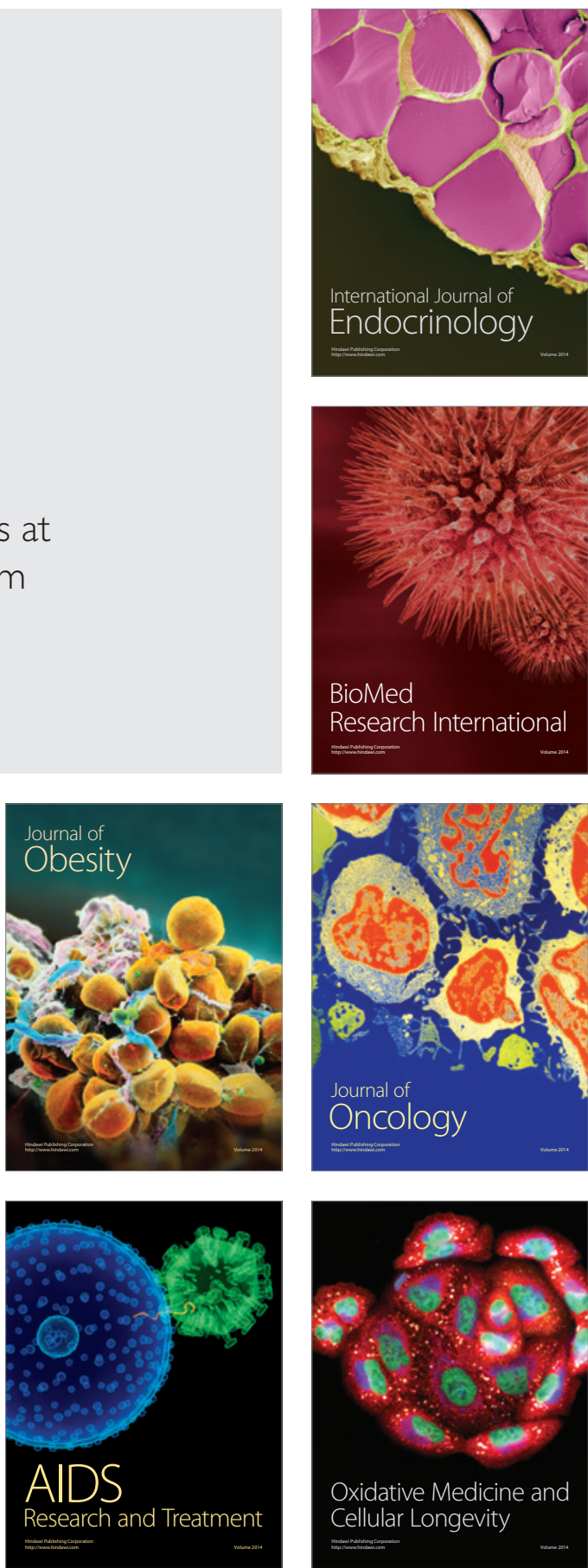